\title{
Toll-Like Receptors 2 and 4 Differentially Regulate the Self-Renewal and Differentiation of Spinal Cord Neural Precursor Cells
}

\section{Marina Sanchez-Petidier}

Neuronal and Tissue Regeneration Laboratory

Consuelo Guerri

Neuropathology Laboratory, Prince Felipe Research Institute

Victoria Moreno-Manzano ( $\nabla$ vmorenom@cipf.es)

Neuronal and Tissue Regeneration Laboratory https://orcid.org/0000-0002-6035-9491

\section{Research}

Keywords: Neural precursor cells, self-renewal, cell differentiation, spinal cord, toll-like receptors, neurogenesis

Posted Date: May 25th, 2021

DOI: https://doi.org/10.21203/rs.3.rs-532850/v1

License: (c) (i) This work is licensed under a Creative Commons Attribution 4.0 International License. Read Full License

Version of Record: A version of this preprint was published at Stem Cell Research \&amp; Therapy on March 21st, 2022. See the published version at https://doi.org/10.1186/s13287-022-02798-z. 


\section{Abstract}

Background: Toll-Like Receptors (TLRs) represent critical effectors in the host defense response against various pathogens; however, their known function during development has also highlighted a potential role in cell fate determination and neural differentiation. While glial cells and neural precursor cells (NPCs) of the spinal cord express both TLR2 and TLR4, their influence on self-renewal and cell differentiation remains incompletely described.

Methods: TLR2, TLR4 knock-out and the wild type mice were employed for spinal cord tissue analysis and NPCs isolation at early post-natal stage. Sox2, FoxJ1 and Ki67 expression among others served to identify the undifferentiated and proliferative NPCs; GFAP, Olig2 and b-III-tubulin markers served to identify astrocytes, oligodendrocytes and neurons respectively after NPC spontaneous differentiation. Multiple comparisons were analyzed using one-way ANOVA, with appropriate corrections such as Tukey's post hoc tests used for comparisons.

Results: We discovered that the deletion of TLR2 or TLR4 significantly reduced the number of Sox2expressing NPCs in the neonatal mouse spinal cord. While TLR2-knockout NPCs displayed enhanced selfrenewal, increased proliferation and apoptosis, and delayed neural differentiation, the absence of TLR4 promoted the neural differentiation of NPCs without affecting proliferation, producing long projecting neurons. TLR4 knock-out NPCs showed significantly higher expression of Neurogenin1, that would be involved in the activation of this neurogenic program by a ligand and microenvironment-independent mechanism. Interestingly, the absence of both TLR2 and TLR4 in NPCs impeded oligodendrocyte precursor cell maturation to a similar degree.

Conclusions: Our data suggest that Toll-Like receptors are needed to maintain Sox2 positive neural progenitors in the spinal cord, however possess distinct regulatory roles in mouse neonatal spinal cord NPCs - while TLR2 and TLR4 play a similar role in oligodendrocytic differentiation, they differentially influence neural differentiation.

\section{Background}

The Toll-like receptor (TLR) family in mammals includes thirteen members - mice and humans express TLR1-9, while mice additionally express TLR11-13, and humans additionally express TLR10 [1]. TLRs respond to a large number of ligands, including pathogen-associated patterns (PAMPs) such as lipopolysaccharide (LPS), nucleic acids, and proteins such as extracellular matrix components (fibrinogen, fibronectin, and hyaluronic acid fragments) or endogenous damage-associated patterns (DAMPs), which are released following injury [2,3]. Interestingly, Drosophila TLRs also recognize neurotrophins such as brain-derived neurotrophic factor (BDNF) and nerve growth factor (NGF) [4]. Following ligand binding, TLRs activate their corresponding signaling components by Myeloid differentiation primary response 88 (MYD88)-dependent and TIR-domain-containing adapter-inducing interferon- $\beta$ (TRIF)-dependent pathways. All TLRs recruit MyD88 except for TLR3, which utilizes TRIF to 
mediate signaling. These signaling pathways have been described for the classical role of TLRs linked to innate immunity that help to orchestrate the immediate and specific adaptive immune response by cytokine and chemokine delivery activating antigen-presenting cells such as macrophages, microglia, or dendritic cells (reviewed in Kawai and Akira [5]).

Interestingly, studies have demonstrated that mammalian TLRs and their Drosophila homologs trigger cell-autonomous processes independent of ligand activation during embryogenesis [3] and adult neurogenesis [6-8]. Constitutive expression of several TLR family members occurs in astrocytes [9], oligodendrocytes [10], neurons [11, 12], and neural precursor cells (NPCs) [13]. Additionally, recent research has suggested a role for TLR2 and TLR4 in supporting neuronal morphogenesis and plasticity under physiological $[3,7]$ and pathological conditions [14-17]. TLR2 forms heterodimers with TLR6 and TLR1 to trigger MyD88-dependent signaling, while TLR4 employs both MyD88-dependent and TRIFdependent signaling pathways $[5,18]$. Rolls et al. studied the function of TLR2 and TLR4 in mouse adult hippocampal NPCs, finding the requirement of TLR4 and MyD88 for astrocytic differentiation and TLR2 in neural differentiation in healthy individuals [7]. TLR4 activation following exposure to LPS, an inflammatory mediator, negatively impacts hippocampal NPC proliferation, suggesting a detrimental effect of TLR4 on neurogenesis [7]; however, TLR4 activation in a model of ischemic stroke-induced NPC proliferation and survival within the subventricular zone promoted neural differentiation of migrating neuroblasts, suggesting a role in endogenous brain repair [19]. Furthermore, Graselli et al. reported a requirement for TLR4 expression in the self-renewal and neuronal/oligodendrocytic differentiation of NPCs from the human fetal brain [20]. Thus, current evidence suggests contrasting roles for TLRs in NPCs, which could derive from ligand-specific activation pathways and/or to cell- and species-specificity.

While we have evidence for the role of TLRs in brain development and morphogenesis, we know little regarding their role in spinal cord NPCs. In the healthy adult human and mouse spinal cord, studies have highlighted TLR2 and TLR4 as the most highly expressed of the TLR family [21, 22], and the significant overexpression of $T L R 2$ and $T L R 4$ following spinal cord injury (SCl) in experimental rodent models [23, 24]. The activation of TLR2 signaling by intrathecal or intramedullary injection of zymosan in the spinal cord produces demyelination, axonal damage, and astrocytic activation [25], which has been attributed to the activation and infiltration of resident microglia and the activation of circulating monocytes, mimicking the inflammatory responses observed after SCI $[25,26]$. However, studies have noted that the absence of TLR2 [24] or TLR4 [27] limits spontaneous regeneration, impairs remyelination, and sustains locomotor deficits after $\mathrm{SCl}$ due, at least in part, to reduced iron metabolism and unbalanced growth factors delivery, which impair oligodendrocyte precursor cell (OPC) maturation [27].

In our new study, we describe the role of TLR2 and TLR4 in the proliferation and differentiation of neonatal spinal cord NPCs. While our results suggest the requirement for both TLR2 and TLR4 to maintain Sox2 positive precursors, they possess distinct roles in neuronal differentiation. While a lack of TLR2 reduce neuronal differentiation and promotes NPC self-renewal, TLR4 loss prompts the increased differentiation of Neurogenin1-expressing neurons. 


\section{Methods}

\section{Study design}

Wild type (WT), $\mathrm{TLR}^{-/-}$and TLR4 ${ }^{-/-}$C57BL/6J mice (kindly provided by Dr. S. Akira, Osaka University, Suita, Japan [28]) were used to comparatively evaluate the influence of both TLRs in NPC self-renewal and cell differentiation. First, spinal cords from at least eight different P4 neonatal mice of each strain, were evaluated by histological analysis. Then, for in vitro experimentation, at least three independent experiments were performed by using a pool of spinal cords of neonates from the same litter, to generate every primary neurosphere-like cultures. NPC identity was evaluated under growth conditions, in the presence of mitogen factors for one day, or in the absence of mitotic factors after one week on culture, in adherent conditions. The percentage of each cell population, astroglial, oligodendrocytes and neurons was evaluated by specific immune staining.

\section{Spinal Cord Tissue Isolation}

Spinal cords from WT, $\mathrm{TLR2}^{-/-}$, or TLR4 ${ }^{-/-}$C57BL/6J mice at the P4 postnatal stage were dissected from cervical to lumbar segments for histological studies or NPC primary cell culture. Spinal cords used for histological analysis were immediately fixed in $4 \%$ paraformaldehyde (PFA) for 4 hours after dissection.

\section{NPC Culture and Treatment}

Following the removal of the overlying meninges and blood vessels, dissected spinal cords were placed in fresh washing medium (WM: DMEM/F12 supplemented with 100 units $/ \mathrm{ml}$ penicillin, $100 \mu \mathrm{g} / \mathrm{ml}$ streptomycin, $5 \mathrm{mM}$ HEPES buffer, $0.125 \% \mathrm{NaHCO}$, and $0.09 \%$ glucose), cut into $1 \mathrm{~mm}^{3}$ pieces, and disaggregated to single-cells in growth medium (GM: NeuroCult ${ }^{\text {TM }}$ Proliferation Medium supplemented with NeuroCult ${ }^{\text {TM }}$ Proliferation Supplement [STEMCELL Technologies, USA] including $20 \mathrm{ng} / \mathrm{ml}$ epidermal growth factor [EGF, Invitrogen], $20 \mathrm{ng} / \mathrm{ml}$ basic fibroblast growth factor [bFGF, Invitrogen], 1X penicillin/streptomycin, and $2 \mu \mathrm{g} / \mathrm{ml}$ heparin [Sigma]) by passing through a $200 \mu \mathrm{l}$ micropipette tip twenty times. NPCs were selected based on their capacity to form neurospheres under non-adherent conditions and then cultured in ultra-low attachment plates in $\mathrm{GM}$ at $37^{\circ} \mathrm{C}$ and $5 \% \mathrm{CO}_{2}$ in a saturated humid atmosphere.

For the quantification of neurosphere number and size, $5 \times 10^{3}$ NPCs were seeded in 96 ultra-low attached/well plates for 48 hours, and then phase-contrast images analyzed using Image J. For proliferation analysis and downstream signaling activation, neurospheres were disaggregated with StemPro Accutase Cell Dissociation Reagent (ThermoFisher) for $5 \mathrm{~min}$ at $37^{\circ} \mathrm{C}$ and seeded onto Matrigel ${ }^{\text {TM }}$ coated coverslips (diluted 1/20 times in DMEM/F12). NPCs cultured at $4 \times 10^{4}$ cells $/ \mathrm{cm}^{2}$ as a monolayer in GM without FGF and EGF were treated with LPS (50 ng/ml, Sigma-Aldrich) for 30 or 60 min.

For spontaneous differentiation analysis, NPCs at $4 \times 10^{4}$ cells $/ \mathrm{cm}^{2}$ were seeded on Matrigel ${ }^{\mathrm{TM}}$ coated coverslips and cultured for seven days. Cells were cultured in GM without EGF and FGF for the first two 
days, after which GM replaced by Differentiation Medium (DM: DMEM/F12 supplemented with 100 units $/ \mathrm{mL}$ penicillin, $100 \mu \mathrm{g} / \mathrm{mL}$ streptomycin, $2 \mathrm{mM}$ L-glutamine, $5 \mathrm{mM}$ HEPES buffer, $0.125 \%$ NaHCO3, $0.6 \%$ glucose, $0.025 \mathrm{mg} / \mathrm{mL}$ insulin, $80 \mu \mathrm{g} / \mathrm{mL}$ apotransferrin, $16 \mathrm{nM}$ progesterone, $60 \mu \mathrm{M}$ putrescine, 24 nM sodium selenite, and $2 \%$ heat-inactivated fetal bovine serum) and maintained for five days.

\section{Population Doubling Level (PDL) Analysis}

PDL analysis was performed in neurosphere-like cultures by seeding NPCs as $5 \times 10^{4}$ cells $/ \mathrm{cm}^{2}$ in ultralow attached plates in GM. Cells were disaggregated and quantified every three days, with the process repeated over fifteen passages. The calculation of PDL used the following formula: $n=\log (2) /(\log U C Y$ $\log \mathrm{I})$, where $\mathrm{n}=$ the final PDL number at the end of a given subculture, UCY = the cell number counted at that time point, $I=$ the cell number seeded to begin that subculture.

\section{Proliferation assay by BrdU Incorporation and Ki67 Immune detection}

NPCs were dissociated and plated on Matrigel ${ }^{\mathrm{Tm}}$ coated coverslips at $4 \times 10^{4}$ cells $/ \mathrm{cm}^{2}$ in GM. 24 hours later, the media was replaced with fresh GM containing $10 \mu \mathrm{M}$ bromodeoxyuridine (BrdU; Roche) for 90 minutes. Cells were then fixed with $4 \%$ PFA for 10 minutes, washed, and incubated with $2 \mathrm{~N} \mathrm{HCl}$ for 20 min at room temperature. The $\mathrm{HCl}$ was then neutralized with $0.1 \mathrm{M}$ sodium borate $(\mathrm{pH} 8.5)$ for 5 min before the double immunofluorescence assay.

\section{Immunofluorescence assay}

After fixation in 4\% PFA, spinal cords were dehydrated and set into paraffin for cross-sectioning into $7 \mu \mathrm{m}$ slices. NPCs cultures were fixed with 4\% PFA for $10 \mathrm{~min}$ at room temperature. Tissue slices were dewaxed and re-hydrated before unmasked using Tris-EDTA Buffer (10 mM Tris Base, 1 mM EDTA Solution, $0.05 \%$ Tween $20, \mathrm{pH} 9.0$ ) at $97^{\circ} \mathrm{C}$ for $25 \mathrm{~min}$ for antigenic retrieval. Permeabilization and blocking steps were performed at the same time using phosphate buffer saline (PBS) containing $0.5 \%$ Triton $x-100,10 \%$ fetal bovine serum, and $5 \%$ horse serum for tissue slices and PBS containing $0.1 \%$ Triton $x-100$ and $3 \%$ normal goat serum for NPCs for 1 hour at room temperature. Next, cells or tissues were incubated overnight at $4{ }^{\circ} \mathrm{C}$ with the primary antibodies at the indicated dilution: a-mouse III-beta-tubulin (1:400, M015013, Neuromics), a-rabbit anti-Olig2 (1:400, AB9610, Millipore), a-chicken Ki67 (1:600, ab15580, Abcam), a-mouse anti-BrdU (1:1000, SIGMA), a-rabbit pH2AX (1:800, 2577, Cell Signaling), a-mouse FOXJ1 (1:200, Invitrogen, 4-9965-82), a-chicken glial fibrillary acidic protein (GFAP; 1:1000, PA1-10004, Thermo Fisher), a-rabbit Sox2 (1:300, ab75179, ABCAM), a-chicken NeuN (1:600. ABN91, Millipore), arabbit anti-TLR2 (1:50, sc-10738, Santa Cruz Biotechnology), a-mouse TLR4 (1:50, sc-293072, Santa Cruz Biotechnology). After washing in PBS, cells were incubated with Goat anti-mouse secondary antibody conjugated with Alexa-Fluor 488, Goat anti-rabbit secondary antibody conjugated with AlexaFluor-555, or Goat anti-chicken conjugated with AlexaFluor-647 (dilution 1:400 in blocking solution, Invitrogen) for 1 hour at room temperature. Finally, nuclei were visualized with 4',6-diamidine-2'-phenylindole dihydrochloride (DAPI; 1:1000, 10 min incubation, Sigma). After additional washes, immune-reactivity was analyzed, and cell images were acquired and analyzed using the Leica IM 5004.0 image-processing 
program (Leica, Bensheim, Germany), confocal microscopy (SP2; Leica), or tissue slice scanner Aperio Versa (Leica Biosystems). The quantification of apoptosis using the levels of pyknotic nuclei stained with DAPI used the identification of labeled nuclei exhibiting a size below the average nucleus size and displaying hyper-condensation of chromatin. GFAP signal intensity was measured using Image $J$ software, and the threshold tool was used to establish the basal signal from WT NPCs. Cell quantification analysis used at least fifteen random fields per group in triplicate in three individual cell cultures. For tissue analysis, all data were normalized to the total evaluated area using Image $\mathrm{J}$ software.

\section{RNA isolation and semi-quantitative RT-PCR}

Total RNA was isolated from NPCs and homogenized spinal cord tissue using TRIzol reagent (Thermo Fisher Scientific) according to the manufacturer's instructions. $1 \mu \mathrm{g}$ of total RNA treated with DNasel (Qiagen) was reverse transcribed using the high-capacity RNA-to-cDNA ${ }^{\mathrm{TM}}$ kit (Applied Biosystems) in a total reaction volume of $20 \mu \mathrm{l}$ through incubation at $37^{\circ} \mathrm{C}$ during $120 \mathrm{~min}$ using random hexamer primers. Specific target primers were designed using Primer-BLAST (NCBI) - sequences, annealing temperature of $60^{\circ} \mathrm{C}$ are detailed in Supplementary Table 1 . A total of $10 \mathrm{ng}$ of CDNA was used for quantitative PCR in a total volume of $10 \mu \mathrm{l}$ using AceQ SYBR qPCR Master Mix (1:2, ThermoFisher) on a LightCycler 480 (Roche, Switzerland). Amplification conditions were determined by each pair of primers to present amplification efficiency close to $100 \%$ and a single peak in melt-curve analyses. Each real-time PCR reaction was performed in triplicate from at least three independent experiments. Peptidylprolyl Isomerase A (PPIA) was used as a housekeeping gene. The log fold change in mRNA expression was calculated from $\Delta \Delta \mathrm{Ct}$ values relative to control samples and the ratio to the housekeeping gene expression ( $\left.2^{\wedge \mathrm{ct} \mathrm{HK}} / 2^{\wedge \text { ctgene }}\right)$ [29].

\section{Western Blot Analysis}

Total protein was extracted from cell cultures using a lysis buffer containing $50 \mathrm{mM} \mathrm{Tris-HCl,} \mathrm{pH} \mathrm{7.5,} 150$ $\mathrm{mM} \mathrm{NaCl}, 0.02 \% \mathrm{NaN3}, 0.1$ SDS, 1 \% NP40, 1 mM EDTA, 2 mg/mL leupeptin, $2 \mathrm{mg} / \mathrm{mL}$ aprotinin, $1 \mathrm{mM}$ phenylmethylsulfonyl fluoride (PMSF), and 1 x Protease Inhibitor Cocktail (Roche Diagnostics, San Diego, CA, USA). The protein concentrations of the supernatant were determined using (BCA) Protein Assay Kit (Thermofisher Scientific). An equal amount of proteins ( $30 \mu \mathrm{g} /$ well) were separated by $10 \%$ SDS-PAGE and transferred to a polyvinylidene fluoride (PVDF) membrane. The membrane was blocked with $1 \%$ bovine serum albumin in Tris buffered-saline with $0.1 \%$ Tween-20 for 1 hour at room temperature and incubated at $4^{\circ} \mathrm{C}$ overnight with the corresponding primary antibody at the indicated dilution: iNOS (1:600, ab15323, ABCAM), IRF1 (1:300, sc-640, Santa Cruz Biotechnology), pERK (1:1000 4370, Cell Signaling), ERK (1:300, sc-271269, Santa Cruz Biotechnology), TLR2 (1:300, sc-21760, Santa Cruz Biotechnology), TLR4 (1:1000, 19811-1-AP, Proteintech), and STAT3 (1:300, sc-482, Santa Cruz Biotechnology).To ensure loading with equal amounts of protein lysates, blots were probed with antibodies against $\alpha$-tubulin or $\beta$ actin (1:10,000 SIGMA). Signal detection was performed with an enhanced chemiluminescence kit (ECL Plus Western blotting detection reagent - GE Healthcare, Piscataway Township, NJ, USA), and bands developed using Amersham Imager 600. Relative protein expression was quantified using the Image Studio Lite software. 


\section{Statistical Analysis}

All experimental data were collected from at least three independent in vitro experiments or up to eight different animals for tissue analysis, and results were reported as the mean \pm the standard error of the mean (S.E.M.) as indicated for each set of data. For the comparisons between two groups of values, the statistical analysis of the results used the Student's t-test for normally distributed data. Results between groups were first assessed for normality using the Shapiro-Wilk test and then analyzed using one-way ANOVA, with appropriate corrections such as Tukey's post hoc tests used for comparisons. Statistical analyses were performed using GraphPad software. Differences were considered significant at * $p<0.05$, $\star * p<0.01, * \star \star p<0.001$ and $* * \star \star p<0.0001$. All statistics and post hoc tests are stated in the text, and corrections for multiple comparisons performed where appropriate.

\section{Results}

\section{TLR2 and TLR4 Expression Maintain the Neural Progenitor Cell Population in the Postnatal Mouse Spinal Cord}

TLR2 and TLR4 displayed similar expression levels in mouse neonatal spinal cord extracts at the mRNA (Fig. 1A) and protein levels (Fig. 1B). We also found constitutive mRNA expression of other TLR family members - while TLR3 showed the highest expression levels, TLRs 6, 8, and 9 displayed lower expression levels than the other tested TLRs (Supplementary Fig. 1). The immune-histochemical analysis of spinal cords provided evidence that most TLR2-expressing cells (orange) co-expressed TLR4 (green), as shown in the representative images (Fig. 1C; white square contains higher magnification of the indicated area shown for each staining).

To further investigate the role of TLR2 and TLR4 in neonatal mouse spinal cord-resident NPCs, we explored the expression of Sox2, the earliest transcription factors expressed in neural stem and progenitor cells [30], playing a key role in specifying early neural lineages and brain development [31]. In the early postnatal WT mouse spinal cord, we observed Sox2 expression within the central canal (CC) (Fig. 1D) in cells homogeneously distributed dorsally and ventrally within the grey matter at the mantle area (Fig. 1D; MT) and within the lining along the cord perimeter in the white matter (PM) (Fig. 1D). Interestingly, the deletion of TLR2 or TLR4 significantly diminished the total number of Sox2-positive cells (Fig. 1E). When we closely inspected Sox2-expressing cells, we distinguished two different expression patterns - nuclear Sox2 expression, corresponding to dividing progenitors NPCs [32] (Fig. 1D, inset *), previously reported as potential oligodendrocyte progenitor cells (OPC) and mature astrocytes [33], and cytoplasmic Sox2 expression, corresponding to migrating and non-dividing neuroblasts [34], co-expressing NeuN (Fig. 1D, inset \#). Our analysis revealed that TLR2 or TLR4 loss only affected the number of cells with nuclear Sox2 expression, assigned as the NPCs (Fig. 1F; solid blue bar), since the total number of OPC (Fig. 1G, left graph) or astrocytes (Fig. 1G, right graph) determined by the expression of Olig2 and GFAP respectively, did not show significant differences. We observed the vast majority of cells with cytoplasmic Sox2 expression located in the grey matter i.e., neuroblasts (Fig. 1H, left panel, blue striped bar) co- 
existing alongside cells with nuclear Sox2 expression (Fig. 1H, left, solid blue bar); however, TLR2 or TLR4 loss failed to impact these Sox2 positive cells. Likewise, all Sox2-positive cells located to the cord perimeter/meningeal zone, [35], displayed nuclear Sox2 expression and here we found a significant impact by the depletion of TLR2 or TLR4 by promoting a significant decrease on the number of this neural precursor population (Fig. 1H, right). Overall, these findings suggest that TLR2 and TLR4 support spinal cord NPC maintenance at early postnatal stages.

An analysis of cell proliferation within the spinal cords of WT and also TLR2 ${ }^{-/}$and TLR4 ${ }^{-/-}$postnatal mice via Ki67 immunostaining failed to find any significant differences (Fig. 1I), suggesting that the decreased number of Sox2-expressing proliferating cells fails to significantly affect overall proliferative activity at the postnatal stage.

We also explored whether the TLR2 or TLR4 expression contributed to the FoxJ1-expressing ependymal precursor cell population. FoxJ1, a transcription factor involved in ciliogenesis [36], is considered a marker of fully differentiated and ciliated ependymal progenitor cells that line the central canal and divide and differentiate at postnatal time points $[37,38]$. However, we failed to find any significant differences in the number of FoxJ1-positive cells in WT, TLR2 ${ }^{-/-}$, and $\mathrm{TLR}^{-/-}$mouse postnatal spinal cords (Fig. $1 \mathrm{~J}$, green). FoxJ1 positive cells co-localized with Sox2 (Fig. $1 \mathrm{~J}$ orange) but not with GFAP (Fig. $1 \mathrm{~J}$, blue), as previously noted [39].

Finally, an analysis of NeuN levels demonstrated a significant reduction in the total number of neurons in $\mathrm{TLR}^{-/-}$but not TLR4 ${ }^{-/-}$mouse neonatal spinal cords than WT mice (Fig. 1K).

Overall, these studies prompted us to undertake a more detailed in vitro analysis of in vitro-expanded NPCs isolated from the spinal cords of WT, TLR2 ${ }^{-/}$, and TLR4 ${ }^{-/-}$mice to decipher the specific roles of these two TLRs in NPC self-renewal and differentiation.

\section{TLR Expression by in vitro-Expanded NPCs Isolated from the Postnatal Mouse Spinal Cord}

We next explored the relative expression of TLR2 and TLR4 by in vitro-expanded NPCs derived from mouse neonatal spinal cords (schematic representation of the NPC in vitro expansion procedure shown in Fig. 2A). We failed to find significant differences in mRNA (Fig. 2B) and protein (Fig. 2D) expression levels for TLR2 and TLR4 in WT NPCs Immunofluorescence analysis confirms that all NPCs co-express for receptors (Fig. 2C). Next, we explored MyD88/TRIF-mediated responses of TLRs in WT NPCs upon stimulation with $50 \mathrm{ng} / \mathrm{ml}$ of LPS for 30 or 60 minutes (Fig. 2E). All three tested downstream mediators of TLR2 and TLR4, induced nitric oxide synthase (iNOS), phosphorylated extracellular signal-regulated kinase (pERK), and interferon regulatory factor 1 (IRF1), displayed maximal activation (as measured by an increase in protein levels for iNOS and IRF1 and phosphorylation of ERK) 30 minutes after stimulation, with a decrease 30 minutes later (Fig. 2E; representative Western blots shown on the left) as previously described in other cell types [40,41]. These data provided evidence for the responsive and functional nature of TLR2 and TLR4 to LPS by in vitroexpanded WT NPCs derived from mouse neonatal spinal cords. 
We also evaluated the expression of the TLR family members in NPCs isolated from the spinal cords of $\mathrm{TLR}^{-/}$and TLR4 ${ }^{-/-}$neonatal mice. Our findings confirmed the lack of TLR2 and TLR4 expression in $\mathrm{TLR}^{-/-}$and TLR4 ${ }^{-/-}$mice, respectively, and additionally demonstrated that TLR2 loss significantly reduced the expression level of $T L R 1, T L R 6, T L R 8$, and $T L R 9$, but not $T L R 3$ and $T L R 4$, and that TLR4 deletion significantly reduced the expression of $T L R 1$ and increased the expression of $T L R 9$ (Fig. 2F).

\section{TLR2, but not TLR4, Regulates the Self-renewal of in vitro-Expanded NPCs Isolated from the Postnatal Mouse Spinal Cord}

We further investigated the role of TLR2 and TLR4 in the self-renewal and proliferation of in vitroexpanded NPCs isolated from the postnatal mouse spinal cord. Isolated and expanded WT, TLR2 ${ }^{-/}$or $\mathrm{TLR}^{-/-}$NPCs all expressed Sox2 (Fig. 3A, representative images in orange; 3D, positive cells quantification) and FoxJ1 (Fig. 3A, representative images in green, $3 \mathrm{C}$, positive cell quantification) to a similar degree (mRNA - Fig. 3B). We again found significant differences regarding Sox2 subcellular location - TLR4 ${ }^{-/}$NPCs displayed a significantly reduced number of cells with nuclear Sox 2 expression compared to TLR2 ${ }^{-1-}$ NPCs or WT NPCs (Fig. 3D). This result agrees with the data found in Fig. $1 \mathrm{H}$ showing a significant reduction of the Sox 2 positive cells at the perimeter SC.

We also evaluated the expression of marker genes preferentially expressed in immature NPCs (Notch1, Sox9, DIx2, NCAM1, Olig1, and PDGFRa) in WT, TLR2 ${ }^{-/}$, or TLR4 ${ }^{-/-}$NPCs to evaluate whether deletion of TLR2 or TLR4 could influence early glial (Sox9, Olig1, PDGFRa) or neuronal determination (Notch1, Dlx2, NCAM1). However, the mRNA expression analysis failed to find any difference in expression for the noted genes suggesting that TLR2 and TLR4 have not significantly influence on these early NPC multilineage markers at this early postnatal stage (Fig. 3E).

We next evaluated the ability of $\mathrm{TLR}^{-{ }^{-}}$and $\mathrm{TLR}^{-/-} \mathrm{NPC}$ to form primary neurospheres to explore their self-renewal capacity [42]. Overall, TLR2 ${ }^{-1-}$ NPCs formed significantly larger but less numerous neurospheres when compared to WT and TLR4 ${ }^{-/-}$NPC-derived neurospheres (Fig. 3F), indicating the preferential formation of primary neurospheres and enhanced self-renewal. Meanwhile, TLR4 ${ }^{-/-}$NPCs formed significantly smaller and more numerous neurospheres (Fig. 3F), indicating the more rapid formation of secondary neurospheres and limited self-renewal.

We also studied proliferation via BrdU incorporation and Ki67 immunostaining in WT, TLR2 ${ }^{-/}$, and TLR4 ${ }^{-/}$ NPCs grown under adherent conditions, finding that only TLR2 deletion significantly increased NPC proliferation (Fig. 3G). Analysis of phospho-H2AX levels, which mark cells undergoing mitotic stress [43], revealed a significantly higher number of positive cells in TLR2 ${ }^{-/-}$NPCs when compared to WT and TLR4 ${ }^{-/-}$ NPCs (Fig. 3H, representative images, right and graph, left). TLR2 ${ }^{-/-}$NPCs also displayed a higher level of cMyc gene expression than TLR2 ${ }^{-/}$NPCs (Fig. 3l, left), indicative of enhanced cell cycle activity [44]. Analysis of p21 expression found significantly lower gene expression levels in TLR4 ${ }^{-/-}$NPCs than WT 
NPCs, suggestive of a potentially deregulated cell cycle (Fig. 3I, right); however, PDL analysis suggested a slow-down in growth for TLR4 ${ }^{-/-}$NPCs only (Fig. 3J).

Interestingly, both TLR2 ${ }^{-/}$and TLR4 ${ }^{-/-}$NPCs exhibited increased apoptosis than WT NPCs. The significant increase in cell death could balance the increased proliferation in TLR2 ${ }^{-/-}$NPCs to explain the lack of PDL differences compared to WT NPCs. As TLR4 ${ }^{-/-}$NPCs did not display higher proliferative rates, the significantly increased apoptosis rate may explain the observed reduction in PDL (Fig. 3K).

Taken together, our data suggest that TLR4 maintains in vitro-expanded NPCs isolated from the postnatal mouse spinal cord in a proliferative and undifferentiated state., while TLR2 expression limits NPC proliferation and self-renewal.

\section{TLR2 and TLR4 in Differentially Contribution to the Formation of Mature Neurons and Glial Cells}

We finally sought to evaluate the contribution of TLR2 or TLR4 to glial or neuronal cell-fate determination by seeding WT, TLR2 ${ }^{-/-}$or TLR4 ${ }^{-/-}$NPCs onto Matrigel ${ }^{\text {TM }}$ coated plates and analyzing comparing their spontaneous differentiation one day (undifferentiated) or seven days (spontaneously differentiated) after growth factor withdrawal, as indicated by the experimental schemes (Fig. 4A, B, top). We failed to encounter any differences in neuronal (B3Tubulin) (Fig. 4A, left panels, green) or astrocytic (GFAP) (Fig. 4A, right panels, red) differentiation of NPCs as a consequence of TLR2 or TLR4 loss after one day (undifferentiated cell condition); however, we encountered significant differences in neuronal (Fig. 4B, left panels) but not astrocytic (Fig. 4B, right panels) differentiation as a consequence of TLR2 or TLR4 loss after seven days. TLR2 ${ }^{-/-}$NPCs displayed a highly significant increase in neuronal differentiation compared to WT and TLR4 ${ }^{-/-}$NPCs, while TLR4 ${ }^{-/-}$NPCs displayed significantly higher neuronal differentiation than WT NPCs (Fig. 4B, left panels).

Interestingly, the $\beta 3$ Tubulin-expressing neuron-like cells exhibited three different morphologies in all three genotypes (Fig. 4C). Type 1 cells are pyramidal-like neurons, with a prominent soma and numerous dendrites (a morphology compatible with mature neuron); Type 2 are immature-like neurons, which are small, with few neurites (a morphology compatible with undifferentiated neurons); and Type 3 cells were bipolar with very long axonal projections (a morphology compatible with mature neurons). TLR2 ${ }^{-/-}$NPCs preferentially differentiated into Type 2 cells (immature neurons) and expressed elevated levels of $D c x$, a marker of very early NPCs (Fig. 4D, upper graph). Meanwhile, TLR4 ${ }^{-/-}$NPCs preferentially differentiated into Type 3 cells (mature neurons) but did not express higher levels of Map2, a marker of mature neurons (Fig. 4D, lower panel), perhaps indicating a transitional stage of maturation.

We next studied the neural cell fate identity of WT, $\mathrm{TLR}^{-/-}$or TLR4 ${ }^{-/-}$NPCs by analyzing Neurogenin 1 expression levels [45]. Both self-renewing and spontaneously differentiated NPCs from TLR4 ${ }^{-/-}$NPCs expressed higher levels of Neurogenin 1) than WT and TLR2 ${ }^{-/-}$NPCs (Fig. 4E), indicating a primed stage of NPCs for neuronal maturation. Overall, TLR2 loss prevented neuronal maturation, while TLR4 loss enhanced neuronal maturation. 
We failed to find any differences in the percentage of astrocytic cells, based on the positive reactivity of GFAP, following analysis of WT, TLR2 ${ }^{-/-}$, or TLR4 ${ }^{-/-}$NPCs at one day or seven days after induced spontaneous differentiation (Fig. 4A, B); however, we discovered a significant increase in GFAP protein expression in TLR4 ${ }^{-/-}$NPCs (Fig. 4F) previously associated to a reactive astrocytic phenotype [46]. Analysis of the Stat3 protein, previously described to be involved in astrocytic reactivity $[47,48]$, revealed a significant increase in TLR4 ${ }^{-/-}$NPCs compared with WT or TLR2 ${ }^{-/-}$NPCs (Fig. 4G), which could explain the reactive phenotypic profile found in the absence of TLR4.

Analysis of oligodendrocyte differentiation in suitable growth-supporting medium found that WT, TLR2 $2^{-/-}$ and TLR4 ${ }^{-/-}$NPCs all expressed similar levels of Olig2, a transcription factor expressed in early to mature stage oligodendrocytes [49] (Fig. 4F, upper panels); however, after seven days of spontaneous differentiation, the absence of TLR2 significantly reduced the generation of Olig2 positive cells from NPCs (Fig. 4F, lower panels). We also found the significantly higher expression of Sox10, a transcription factor expressed in early OPCs [50], and the significantly lower expression of NG2, a factor expressed by mature OPCs [51], in the absence of TLR2 or TLR4. Overall, this data also suggests a critical role for TLR2 and TLR4 in oligodendrocyte maturation.

\section{Discussion}

In addition to their ability to respond to the innate immune system, TLRs play fundamental roles in cell fate and cell maturation of NPCs in the developing and adult mammalian brain, with studies suggesting the implication of both TLR2 and TLR4 [3]. In our exploration of TLR expression in the neonatal mouse spinal cord, we discovered that TLR2 and TLR4 help to maintain the NPC population and influence cell fate determination in a cell-autonomous manner. Based on the spontaneous capacity of the NPCs lacking of TLR2 or TLR4 and also based on their expression of Neurogenin 1, we propose that TLR2 promotes and TLR4 restricts neural maturation, respectively.

During early postnatal stages, at a point when the mouse spinal cord remain incompletely differentiated, Sox2-expressing NPCs exist throughout the spinal cord (in the grey and white matter) lining the meninges and in the ependymal canal, where they co-express FoxJ1, representing the neurogenic niches [38]. NPCs continuously express Sox 2 until adulthood to maintain self-renewal capacity by regulating the expression of crucial genes; however, a shift of Sox2 location from the nucleus to the cytoplasm promotes their differentiation $[52,53]$. Hyper-acetylation of Sox 2 prompts translocation into the cytoplasm and then the differentiation of NPCs [54]. We found that NPCs with nuclear Sox2 expression primarily located to the grey matter of the spinal cord and co-expressed the neuronal marker NeuN but lacked Ki67 expression (data not shown), indicating the existence of a transitional differentiation stage. Despite a significant reduction in the total number of Sox2-positive cells following the loss of TLR2 or TLR4, the proportion of NPCs with cytoplasmic Sox2 remained unchanged, indicating a relevant and restrictive influence of both receptors at the undifferentiated stages when Sox 2 retains its transcriptional activity. Since Sox 2 positive precursors are actively dividing, we also expected a reduction in the acutely number of mitotic cells, 
positive for ki67, however, neither TLR2 or TLR4 deletion significantly influenced over a global proliferating rate that deserves further investigation. Previous studies indicated that TLR4 deletion increases the proliferation of retinal precursors [55] while TLR2 deletion failed to modify mitotic rates in the hippocampus [7], therefore suggesting the existence of specific mechanisms for the TLR2 and TLR4mediated regulation of NPCs in the postnatal mouse spinal cord.

To further evaluate the novel functions of TLR2 and TLR4 within the neurogenic niche of the spinal cord, we isolated and in vitro-expanded NPCs. NPCs from postnatal or adult stages that form neurosphere 3Dlike cultures retain their self-renewal and multipotent capacity [42], even taking the form of a pinwheel-like cytoarchitectural structure that mimics the in vivo organization of the endogenous neurogenic niche [56]. NPCs within neurosphere-like cultures maintain the expression of crucial markers such as Sox2 and FoxJ1 and self-renewal/cell differentiation capacities. Our analysis in neurosphere-like cultures found evidence that TLR4- but not TLR2 ${ }^{-/-}$NPCs reproduced in vivo findings, showing a significant decrease in the percentage of nuclear Sox2-expressing cells. Additionally, a reduction in the proliferation of TLR4 ${ }^{-/-}$ NPCs suggested a restrictive self-renewal profile, validating the critical role of TLR4 in maintaining mouse neonatal spinal cord NPCs in an undifferentiated state. However, TLR2 ${ }^{-/-}$NPCs, which formed bigger primary neurospheres and possessed higher mitotic activity, also displayed significantly higher rates of apoptosis, suggesting enhanced self-renewal. The increased level of apoptosis could explain the reduced percentage of Sox2-positive spinal cord NPCs in the absence of TLR2, which would not be compensated by the significant, although modest, increase in mitosis. In a related study, TLR2 deletion in the adult hippocampus under physiological conditions affected cell-fate decisions but not self-renewal, whereas TLR4 loss affected both proliferation and differentiation of NPCs [7].

Downstream TLR signaling, activated by distinct ligands for each different member, displays different consequences on NPC maturation. For instance, immortalized human NPCs expressing TLR4 respond to LPS-mediated antagonism by significantly decreasing proliferation and survival, indicating that TLR4 contributes to self-renewing upon exogenous and endogenous activation [20]. The same study linked TLR4 expression and activation to neuronal and oligodendroglial differentiation [20], in agreement with our findings in mouse NPCs. Also, in agreement with previous reports [20,57], we linked TLR2 and TLR4 expression to OPC maturation in physiological conditions. NPCs lacking TLR2 or TLR4 retain an undifferentiated stage by expressing elevated levels of Sox10, which is associated with early oligodendrocyte commitment, and lower levels of NG2, which is expressed at later stages during OPC maturation.

We know little regarding the precise cellular and molecular mechanisms involving TLRs in the interplay between immune cells and NPCs. Complex cell-autonomous and non-cell-autonomous mechanisms operate concomitantly with the involvement of TLR-dependent signaling $[58,59]$. While studies have demonstrated that immune-deficient mice, with limited TLR activation, show impaired neurogenesis and that specific T helper cells, TLR activators, promote neurogenesis in the adult hippocampus [60], TLR4 has been linked to spontaneous oligodendrocyte maturation and remyelination after damage in an inflammatory environment, such as after SCI [27]. Well-orchestrated adaptive immune responses can also 
support/induce oligodendrogenesis and neurogenesis. Microglia activated in response to Interleukin-4 support oligodendrogenesis, whereas interferon (IFN)-y-activated microglia showed a bias towards neurogenesis [61]. Of note, neuron-enriched cultures promote oligodendrogenesis rather than astrogliogenesis, indicating that neurons, and not exclusively immune cells within the neurogenic niche, create the local microenvironment [62]. Here we show that TLR4 deletion in NPCs promotes neurogenesis during spontaneous differentiation; however, whether cell-cell interactions or the release of specific factors contributes to cell fate maturation remains unknown.

The Jak/Stat axis plays a pivotal role in NPC astrocytic differentiation, reactivity [63], and survival [48] and neuronal differentiation [64]. At early developmental stages, Jak/Stat pathway components drives the neurogenic phase, when astrocytic genes are still silent [64]. Proneural proteins such as Neurog1, a bHLH protein, play an essential role in this process, as their deletion causes precocious astrocyte differentiation and limited neural cell fate [65]. We found that the absence of TLR4 prompts an increase in both Neurog1 and Stat3 expression, which prompted neural differentiation and astrocytic activation. The STAT3-mediated neurogenic-to-astrogenic fate switch has been previously described in cultured NPCs [66] (reinforcing the idea of cell-intrinsic programs [67]).

\section{Conclusions}

we found a ligand and microenvironment-independent program that regulates neural precursor cells population maintenance and the neural differentiation at the neonatal stages involving TLR2 and TLR4 depending-signaling and the constitutive expression of Neurogenin1. Thus, TLR signaling regulation could represent a promising avenue to increase cellular plasticity and promote neural differentiation in the spinal cord.

\section{Abbreviations}

BCA: Bicinchoninic Acid;BDNF: Brain-derived neurotrophic factor;bFGF: basic Fibroblast growth factor; BrdU: Bromodeoxyuridine; DAMP: Damage-associated patterns;DAPI: 4',6-diamidine-2'-phenylindole dihydrochloride; DM: Differentiation medium; EDTA: Ethylene diamine tetra-acetic acid; EGF: Epidermal growth factor; ERK: Extracellular signal-regulated kinases; GFAP: Glial fibrillary acidic protein; GM: Growth medium; IFN: Interferon; iNOS: Inducible nitric oxide synthase; IRF: Interferon regulatory factor; LPS: Lipopolysaccharide; MAP: Microtubule-associated protein; MYD88: Myeloid differentiation primary response 88; NCAM: Neural cell adhesion molecule; NGF: Nerve growth factor; NPC: Neural Progenitor Cell; OPC: Oligodendrocyte precursor cell; PAMP: Pathogen-associated patterns; PBS: Phosphate buffer saline; PDGFR: Platelet-derived growth factor receptor; PDL: Population Doubling Level; PFA:

Paraformaldehyde; PMSF: Phenylmethylsulfonyl fluoride; PPIA: Peptidylprolyl Isomerase A; RT-PCR: RealTime Polymerase Chain Reaction; SCl: Spinal cord injury; SEM: Standard error of the mean; SOX: SRY-Box Transcription Factor; STAT: signal transducer and activator of transcription; TLR: Toll-like Receptor; TRIF: TIR-domain-containing adapter-inducing interferon; WM: Washing medium; WT: wild type 


\section{Declarations}

Ethics approval: All animal experiments were undertaken in accordance with guidelines established by the European Communities Council Directive (86/609/ECC) and the Spanish Royal Decree 1201/2005. The experimental procedure was approved by the institutional Animal Care and Use Committee with the reference number 2018/VSC/PEA/0056. All animals were managed by professionally trained staff. Water and food were provided ad-libitum throughout the experimental timeframes with general aspect, behavior, and body weight evaluated daily to ensure animal welfare.

Consent for publication: Not applicable

Availability of data and materials: The datasets used and/or analyzed during the current study are available from the corresponding author on reasonable request.

Competing of Interest: the authors declare that they have no competing interest.

Funding: This research was funded by FEDER/Ministerio de Ciencia e Innovación - Agencia Estatal de Investigación “RTI2018-095872-B-C21/ERDF”, Fundació Marató TV3 2017/refs.20172230, 20172231 and 20172110, Fundación Valenciana Corre per mi; Agencia Valenciana de Innovación (AVI) INNVAL10/19/047 and MINECO/FEDER, UE; Fondo Europeo de Desarrollo Regional (FEDER) incluido en el Programa Operativo FEDER de la Comunidad Valenciana 2014-2020).

Acknowledgements: The authors would like to thank Maravillas Mellado for her excellent technical support.

Author's contributions: MSP: Collection and/or assembly of data, Data analysis and interpretation, Manuscript writing; CG: Final approval of the manuscript, Financial support; VMM: Conception and design, Manuscript writing, Data analysis and interpretation, Final approval of the manuscript, Financial support

\section{References}

1. Roach JC, Glusman G, Rowen L, Kaur A, Purcell MK, Smith KD, et al. The evolution of vertebrate Tolllike receptors. Proc Natl Acad Sci U S A. 2005;102(27):9577-82. doi:10.1073/pnas.0502272102.

2. Akira S, Uematsu S, Takeuchi O. Pathogen recognition and innate immunity. Cell. 2006;124(4):783801. doi:10.1016/j.cell.2006.02.015.

3. Anthoney N, Foldi I, Hidalgo A. Toll and Toll-like receptor signalling in development. Development. 2018;145(9). doi:10.1242/dev.156018.

4. Foldi I, Anthoney N, Harrison N, Gangloff M, Verstak B, Nallasivan MP, et al. Three-tier regulation of cell number plasticity by neurotrophins and Tolls in Drosophila. J Cell Biol. 2017;216(5):1421-38. doi:10.1083/jcb.201607098. 
5. Kawai T, Akira S. TLR signaling. Semin Immunol. 2007;19(1):24-32. doi:10.1016/j.smim.2006.12.004.

6. Okun E, Griffioen KJ, Mattson MP. Toll-like receptor signaling in neural plasticity and disease. Trends Neurosci. 2011;34(5):269-81. doi:10.1016/j.tins.2011.02.005.

7. Rolls A, Shechter R, London A, Ziv Y, Ronen A, Levy R, et al. Toll-like receptors modulate adult hippocampal neurogenesis. Nat Cell Biol. 2007;9(9):1081-8. doi:10.1038/ncb1629.

8. Martino G, Pluchino S, Bonfanti L, Schwartz M. Brain regeneration in physiology and pathology: the immune signature driving therapeutic plasticity of neural stem cells. Physiol Rev. 2011;91(4):1281304. doi:10.1152/physrev.00032.2010.

9. Bowman CC, Rasley A, Tranguch SL, Marriott I. Cultured astrocytes express toll-like receptors for bacterial products. Glia. 2003;43(3):281-91. doi:10.1002/glia.10256.

10. Aravalli RN, Peterson PK, Lokensgard JR. Toll-like receptors in defense and damage of the central nervous system. J Neuroimmune Pharmacol. 2007;2(4):297-312. doi:10.1007/s11481-007-9071-5.

11. Sauter MM, Kolb AW, Brandt CR. Toll-like receptors 4, 5, 6 and 7 are constitutively expressed in nonhuman primate retinal neurons. J Neuroimmunol. 2018;322:26-35.

doi:10.1016/j.jneuroim.2018.06.007.

12. Chen $C Y$, Shih YC, Hung YF, Hsueh YP. Beyond defense: regulation of neuronal morphogenesis and brain functions via Toll-like receptors. J Biomed Sci. 2019;26(1):90. doi:10.1186/s12929-019-0584-Z.

13. Liu HY, Chen $\mathrm{CY}$, Hsueh YP. Innate immune responses regulate morphogenesis and degeneration: roles of Toll-like receptors and Sarm1 in neurons. Neurosci Bull. 2014;30(4):645-54. doi:10.1007/s12264-014-1445-5.

14. Ziv Y, Ron N, Butovsky O, Landa G, Sudai E, Greenberg N, et al. Immune cells contribute to the maintenance of neurogenesis and spatial learning abilities in adulthood. Nat Neurosci. 2006;9(2):268-75. doi:10.1038/nn1629.

15. Hsieh CH, Rau CS, Kuo PJ, Liu SH, Wu CJ, Lu TH, et al. Knockout of toll-like receptor impairs nerve regeneration after a crush injury. Oncotarget. 2017;8(46):80741-56. doi:10.18632/oncotarget.20206.

16. Manivannan S, Marei O, Elalfy O, Zaben M. Neurogenesis after traumatic brain injury - The complex role of HMGB1 and neuroinflammation. Neuropharmacology. 2020;183:108400. doi:10.1016/j.neuropharm.2020.108400.

17. Ulrich H, do Nascimento IC, Bocsi J, Tarnok A. Immunomodulation in stem cell differentiation into neurons and brain repair. Stem Cell Rev Rep. 2015;11(3):474-86. doi:10.1007/s12015-014-9556-6.

18. Brennan JJ, Gilmore TD. Evolutionary Origins of Toll-like Receptor Signaling. Mol Biol Evol. 2018;35(7):1576-87. doi:10.1093/molbev/msy050.

19. Palma-Tortosa S, Hurtado O, Pradillo JM, Ferreras-Martin R, Garcia-Yebenes I, Garcia-Culebras A, et al. Toll-like receptor 4 regulates subventricular zone proliferation and neuroblast migration after experimental stroke. Brain Behav Immun. 2019;80:573-82. doi:10.1016/j.bbi.2019.05.002. 
20. Grasselli C, Ferrari D, Zalfa C, Soncini M, Mazzoccoli G, Facchini FA, et al. Toll-like receptor 4 modulation influences human neural stem cell proliferation and differentiation. Cell Death Dis. 2018;9(3):280. doi:10.1038/s41419-017-0139-8.

21. Nishimura M, Naito $S$. Tissue-specific mRNA expression profiles of human toll-like receptors and related genes. Biol Pharm Bull. 2005;28(5):886-92. doi:10.1248/bpb.28.886.

22. Adhikary S, Li H, Heller J, Skarica M, Zhang M, Ganea D, et al. Modulation of inflammatory responses by a cannabinoid-2-selective agonist after spinal cord injury. J Neurotrauma. 2011;28(12):2417-27. doi:10.1089/neu.2011.1853.

23. Heiman A, Pallottie A, Heary RF, Elkabes S. Toll-like receptors in central nervous system injury and disease: a focus on the spinal cord. Brain Behav Immun. 2014;42:232-45. doi:10.1016/j.bbi.2014.06.203.

24. Kigerl KA, Lai W, Rivest S, Hart RP, Satoskar AR, Popovich PG. Toll-like receptor (TLR)-2 and TLR-4 regulate inflammation, gliosis, and myelin sparing after spinal cord injury. J Neurochem. 2007;102(1):37-50. doi:10.1111/j.1471-4159.2007.04524.x.

25. Popovich PG, Guan Z, McGaughy V, Fisher L, Hickey WF, Basso DM. The neuropathological and behavioral consequences of intraspinal microglial/macrophage activation. J Neuropathol Exp Neurol. 2002;61(7):623-33. doi:10.1093/jnen/61.7.623.

26. Schonberg DL, Popovich PG, McTigue DM. Oligodendrocyte generation is differentially influenced by toll-like receptor (TLR) 2 and TLR4-mediated intraspinal macrophage activation. J Neuropathol Exp Neurol. 2007;66(12):1124-35. doi:10.1097/nen.0b013e31815c2530.

27. Church JS, Kigerl KA, Lerch JK, Popovich PG, McTigue DM. TLR4 Deficiency Impairs Oligodendrocyte Formation in the Injured Spinal Cord. J Neurosci. 2016;36(23):6352-64. doi:10.1523/JNEUROSCI.0353-16.2016.

28. Akira S, Takeda K, Kaisho T. Toll-like receptors: critical proteins linking innate and acquired immunity. Nat Immunol. 2001;2(8):675-80. doi:10.1038/90609.

29. Livak KJ, Schmittgen TD. Analysis of relative gene expression data using real-time quantitative PCR and the 2(-Delta Delta C(T)) Method. Methods. 2001;25(4):402-8. doi:10.1006/meth.2001.1262.

30. Avilion AA, Nicolis SK, Pevny LH, Perez L, Vivian N, Lovell-Badge R. Multipotent cell lineages in early mouse development depend on SOX2 function. Genes Dev. 2003;17(1):126-40. doi:10.1101/gad.224503.

31. Bylund M, Andersson E, Novitch BG, Muhr J. Vertebrate neurogenesis is counteracted by Sox1-3 activity. Nat Neurosci. 2003;6(11):1162-8. doi:10.1038/nn1131.

32. Kim JB, Zaehres H, Wu G, Gentile L, Ko K, Sebastiano V, et al. Pluripotent stem cells induced from adult neural stem cells by reprogramming with two factors. Nature. 2008;454(7204):646-50. doi:10.1038/nature07061.

33. Zhao C, Ma D, Zawadzka M, Fancy SP, Elis-Williams L, Bouvier G, et al. Sox2 Sustains Recruitment of Oligodendrocyte Progenitor Cells following CNS Demyelination and Primes Them for Differentiation during Remyelination. J Neurosci. 2015;35(33):11482-99. doi:10.1523/JNEUROSCI.3655-14.2015. 
34. Ferri AL, Cavallaro M, Braida D, Di Cristofano A, Canta A, Vezzani A, et al. Sox2 deficiency causes neurodegeneration and impaired neurogenesis in the adult mouse brain. Development. 2004;131(15):3805-19. doi:10.1242/dev.01204.

35. Decimo I, Fumagalli G, Berton V, Krampera M, Bifari F. Meninges: from protective membrane to stem cell niche. Am J Stem Cells. 2012;1(2):92-105.

36. Yu X, Ng CP, Habacher H, Roy S. Foxj1 transcription factors are master regulators of the motile ciliogenic program. Nat Genet. 2008;40(12):1445-53. doi:10.1038/ng.263.

37. Spassky N, Merkle FT, Flames N, Tramontin AD, Garcia-Verdugo JM, Alvarez-Buylla A. Adult ependymal cells are postmitotic and are derived from radial glial cells during embryogenesis. $J$ Neurosci. 2005;25(1):10-8. doi:10.1523/JNEUROSCI.1108-04.2005.

38. Meletis K, Barnabe-Heider F, Carlen M, Evergren E, Tomilin N, Shupliakov O, et al. Spinal cord injury reveals multilineage differentiation of ependymal cells. PLoS Biol. 2008;6(7):e182. doi:10.1371/journal.pbio.0060182.

39. Moreno-Manzano V. Ependymal cells in the spinal cord as neuronal progenitors. Curr Opin Pharmacol. 2020;50:82-7. doi:10.1016/j.coph.2019.11.008.

40. Fernandez-Lizarbe S, Montesinos J, Guerri C. Ethanol induces TLR4/TLR2 association, triggering an inflammatory response in microglial cells. J Neurochem. 2013;126(2):261-73. doi:10.1111/jnc.12276.

41. Gong W, Li J, Chen W, Feng F, Deng Y. Resveratrol Inhibits Lipopolysaccharide-Induced Extracellular Matrix Accumulation and Inflammation in Rat Glomerular Mesangial Cells by SphK1/S1P2/NFkappaB Pathway. Diabetes Metab Syndr Obes. 2020;13:4495-505. doi:10.2147/DMSO.S278267.

42. Slovinska L, Szekiova E, Blasko J, Devaux S, Salzet M, Cizkova D. Comparison of dynamic behavior and maturation of neural multipotent cells derived from different spinal cord developmental stages: an in vitro study. Acta Neurobiol Exp (Wars). 2015;75(1):107-14.

43. Ropolo M, Daga A, Griffero F, Foresta M, Casartelli G, Zunino A, et al. Comparative analysis of DNA repair in stem and nonstem glioma cell cultures. Mol Cancer Res. 2009;7(3):383-92. doi:10.1158/1541-7786.MCR-08-0409.

44. Kelly K, Cochran B, Stiles C, Leder P. The regulation of c-myc by growth signals. Curr Top Microbiol Immunol. 1984;113:117-26. doi:10.1007/978-3-642-69860-6_22.

45. Ma Q, Sommer L, Cserjesi P, Anderson DJ. Mash1 and neurogenin1 expression patterns define complementary domains of neuroepithelium in the developing CNS and are correlated with regions expressing notch ligands. J Neurosci. 1997;17(10):3644-52.

46. Pekny M, Pekna M. Astrocyte reactivity and reactive astrogliosis: costs and benefits. Physiol Rev. 2014;94(4):1077-98. doi:10.1152/physrev.00041.2013.

47. Kang MK, Kang SK. Interleukin-6 induces proliferation in adult spinal cord-derived neural progenitors via the JAK2/STAT3 pathway with EGF-induced MAPK phosphorylation. Cell Prolif. 2008;41(3):37792. doi:10.1111/j.1365-2184.2008.00537.x. 
48. Levine J, Kwon E, Paez P, Yan W, Czerwieniec G, Loo JA, et al. Traumatically injured astrocytes release a proteomic signature modulated by STAT3-dependent cell survival. Glia. 2016;64(5):668-94. doi:10.1002/glia.22953.

49. Lu QR, Park JK, Noll E, Chan JA, Alberta J, Yuk D, et al. Oligodendrocyte lineage genes (OLIG) as molecular markers for human glial brain tumors. Proc Natl Acad Sci U S A. 2001;98(19):10851-6. doi:10.1073/pnas.181340798.

50. Stolt CC, Rehberg S, Ader M, Lommes P, Riethmacher D, Schachner M, et al. Terminal differentiation of myelin-forming oligodendrocytes depends on the transcription factor Sox10. Genes Dev. 2002;16(2):165-70. doi:10.1101/gad.215802.

51. Polito A, Reynolds R. NG2-expressing cells as oligodendrocyte progenitors in the normal and demyelinated adult central nervous system. J Anat. 2005;207(6):707-16. doi:10.1111/j.14697580.2005.00454.x.

52. Zhao S, Nichols J, Smith AG, Li M. SoxB transcription factors specify neuroectodermal lineage choice in ES cells. Mol Cell Neurosci. 2004;27(3):332-42. doi:10.1016/j.mcn.2004.08.002.

53. Takemoto T, Uchikawa M, Yoshida M, Bell DM, Lovell-Badge R, Papaioannou VE, et al. Tbx6dependent Sox2 regulation determines neural or mesodermal fate in axial stem cells. Nature. 2011;470(7334):394-8. doi:10.1038/nature09729.

54. Baltus GA, Kowalski MP, Zhai H, Tutter AV, Quinn D, Wall D, et al. Acetylation of sox2 induces its nuclear export in embryonic stem cells. Stem Cells. 2009;27(9):2175-84. doi:10.1002/stem.168.

55. Shechter R, Ronen A, Rolls A, London A, Bakalash S, Young MJ, et al. Toll-like receptor 4 restricts retinal progenitor cell proliferation. J Cell Biol. 2008;183(3):393-400. doi:10.1083/jcb.200804010.

56. Rodriguez-Jimenez FJ, Clemente E, Moreno-Manzano V, Erceg S. Organized Neurogenic-Niche-Like Pinwheel Structures Discovered in Spinal Cord Tissue-Derived Neurospheres. Front Cell Dev Biol. 2019;7:334. doi:10.3389/fcell.2019.00334.

57. Bsibsi M, Nomden A, van Noort JM, Baron W. Toll-like receptors 2 and 3 agonists differentially affect oligodendrocyte survival, differentiation, and myelin membrane formation. J Neurosci Res. 2012;90(2):388-98. doi:10.1002/jnr.22767.

58. Fan LW, Pang Y. Dysregulation of neurogenesis by neuroinflammation: key differences in neurodevelopmental and neurological disorders. Neural Regen Res. 2017;12(3):366-71. doi:10.4103/1673-5374.202926.

59. Kokaia Z, Martino G, Schwartz M, Lindvall O. Cross-talk between neural stem cells and immune cells: the key to better brain repair? Nat Neurosci. 2012;15(8):1078-87. doi:10.1038/nn.3163.

60. Song D, Qi F, Liu S, Tang Z, Duan J, Yao Z. The adoptive transfer of BCG-induced T lymphocytes contributes to hippocampal cell proliferation and tempers anxiety-like behavior in immune deficient mice. PLoS One. 2020;15(4):e0225874. doi:10.1371/journal.pone.0225874.

61. Butovsky O, Ziv Y, Schwartz A, Landa G, Talpalar AE, Pluchino S, et al. Microglia activated by IL-4 or IFN-gamma differentially induce neurogenesis and oligodendrogenesis from adult stem/progenitor cells. Mol Cell Neurosci. 2006;31(1):149-60. doi:10.1016/j.mcn.2005.10.006. 
62. Chien $\mathrm{CH}$, Lee MJ, Liou HC, Liou HH, Fu WM. Microglia-Derived Cytokines/Chemokines Are Involved in the Enhancement of LPS-Induced Loss of Nigrostriatal Dopaminergic Neurons in DJ-1 Knockout Mice. PLoS One. 2016;11(3):e0151569. doi:10.1371/journal.pone.0151569.

63. Bonni A, Sun Y, Nadal-Vicens M, Bhatt A, Frank DA, Rozovsky I, et al. Regulation of gliogenesis in the central nervous system by the JAK-STAT signaling pathway. Science. 1997;278(5337):477-83. doi:10.1126/science.278.5337.477.

64. Molne M, Studer L, Tabar V, Ting YT, Eiden MV, McKay RD. Early cortical precursors do not undergo LIF-mediated astrocytic differentiation. J Neurosci Res. 2000;59(3):301-11. doi: 10.1002/(sici)10974547(20000201)59:3<301::aid-jnr3>3.0.co;2-h.

65. Tomita K, Moriyoshi K, Nakanishi S, Guillemot F, Kageyama R. Mammalian achaete-scute and atonal homologs regulate neuronal versus glial fate determination in the central nervous system. EMBO J. 2000;19(20):5460-72. doi:10.1093/emboj/19.20.5460.

66. Qian X, Shen Q, Goderie SK, He W, Capela A, Davis AA, et al. Timing of CNS cell generation: a programmed sequence of neuron and glial cell production from isolated murine cortical stem cells. Neuron. 2000;28(1):69-80. doi:10.1016/s0896-6273(00)00086-6.

67. Lee HC, Tan KL, Cheah PS, Ling KH. Potential Role of JAK-STAT Signaling Pathway in the Neurogenic-to-Gliogenic Shift in Down Syndrome Brain. Neural Plast. 2016;2016:7434191. doi:10.1155/2016/7434191.

\section{Figures}



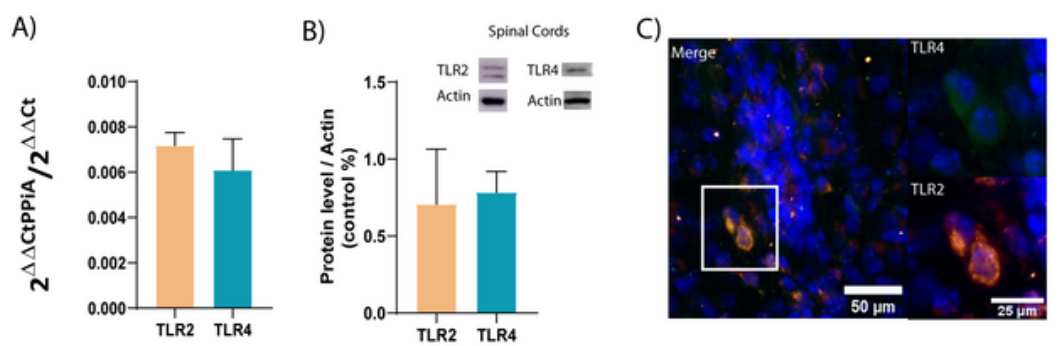

D)
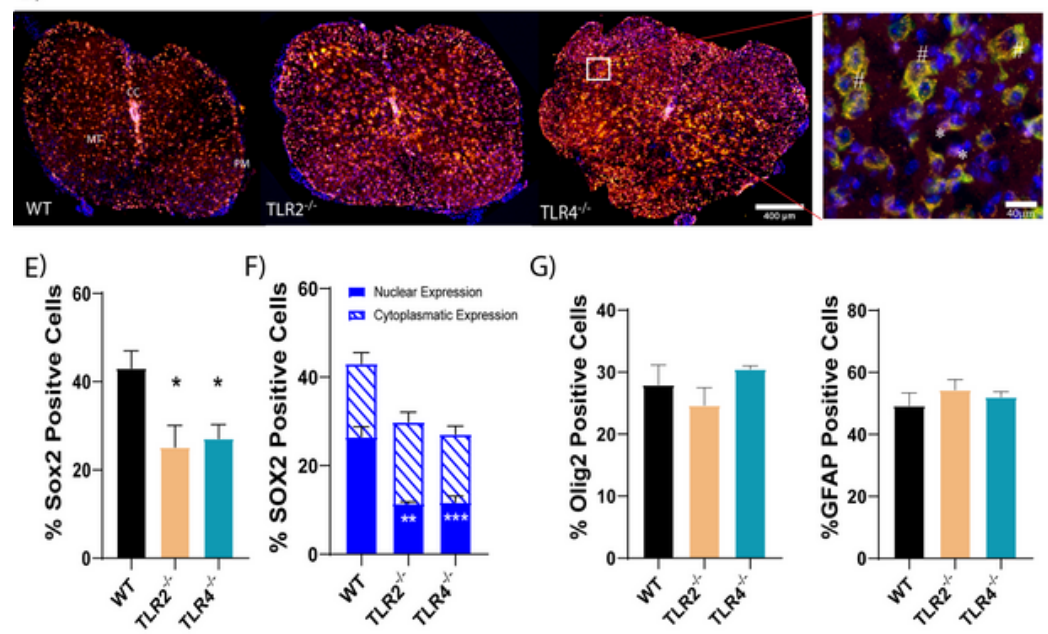

G)

H)
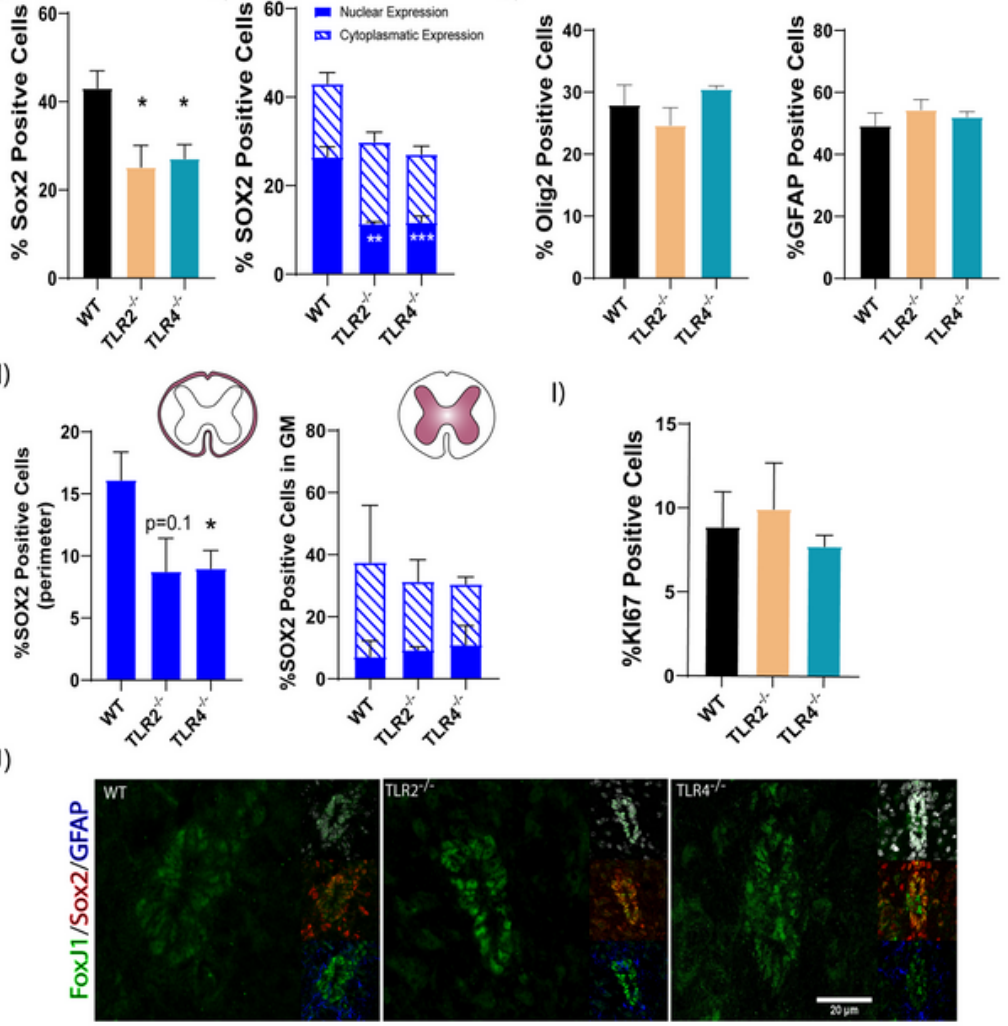

K)

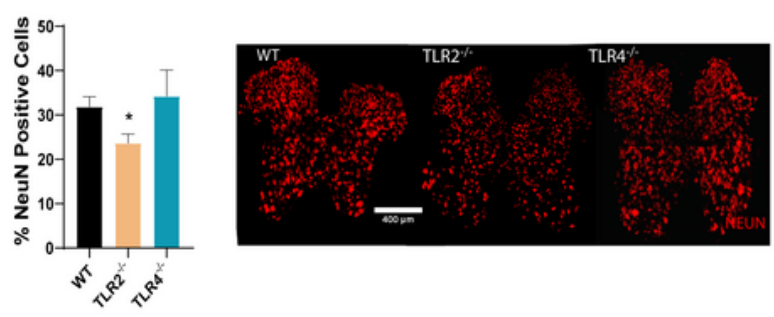

\section{Figure 1}

Analysis of Spinal Cord NPCs from WT and TLR2 and TLR4 Knockout Mice. (A) Gene expression analysis of TLR2 and TLR4 in the spinal cord tissue of WT mice; (B) Representative Western blots and the overall protein levels of TLR2 and TLR4 from WT spinal cord tissue. $\beta$-actin was used as a total protein loading control; (C) Representative image showing double immunoreactivity (merge) for TLR2 (orange) or TLR4 (green) and DAPI (blue -for nuclei counterstaining) in spinal cord coronal slices. A magnified view of the 
indicated area with a white square for each staining; (D) Left panels: Representative immunofluorescence images of SOX2 (orange) and DAPI (blue) in spinal cord coronal sections from WT, TLR2-/- and TLR4 -/mice; Right panel: magnified view of the area indicated with a white square to show the nuclear $\left(^{*}\right)$ or cytoplasmic (\#) sub-cellular expression of Sox2 with cytoplasmic co-localization with NeuN (green). (E) Quantification of Sox2-positive cells expressed as a percentage of the total number of cells. (F) Quantification of nuclear (full) or cytoplasmic (striped) Sox2-expressing cells as a percentage of the total number of cells in the entire spinal cord area. (G) Left graph: Quantification of Olig2-positive cells expressed as a percentage of the total number of cells in spinal cord coronal sections; Right graph: GFAPpositive cells expressed as a percentage of the total number of cells in spinal cord coronal sections. $(H)$ Quantification of nuclear (full) or cytoplasmic (striped) Sox2-expressing cells as a percentage of the total number of cells in the grey matter (left) and white matter at the PM (right). (J) Quantification of \% of cells positive for KI67. (I) Representative immunostaining images of the CC of WT, TLR2-/- or TLR4-/- mouse spinal cords. For each genotype - Main large image - FOXJ (green); Small upper right image DAPI/FOXJ1 merge (white); Small middle right - SOX2/FOXJ1 merge (red); GFAP/FOXJ1 merge (blue). (K) (Left) Quantification of NeuN-positive cells expressed as a percentage of the total number of cells in spinal cord coronal sections. (Right) Representative images of NeuN staining in WT, TLR2-/- or TLR4-/spinal cords. Data shown as mean \pm SEM. Results assessed for normality using the Shapiro-Wilk test and one-way ANOVA with Tukey post hoc test; ${ }^{\star} p<0.05$, ${ }^{\star \star} p<0.01$ or ${ }^{\star \star \star} p<0.001$ vs. WT. 
A)

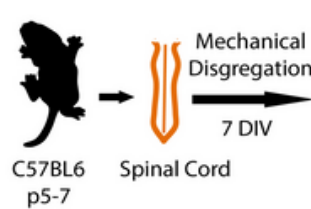

C)

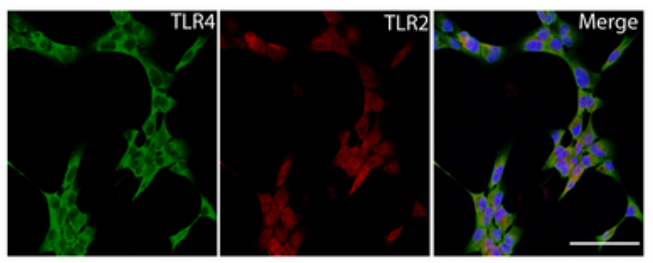

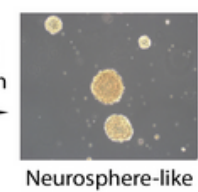

3D culture
B)

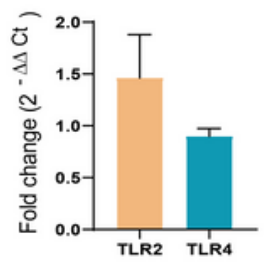

E)

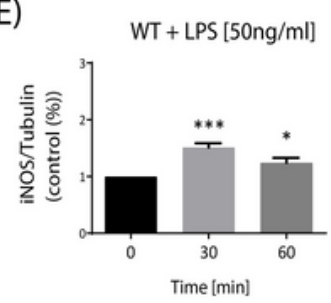

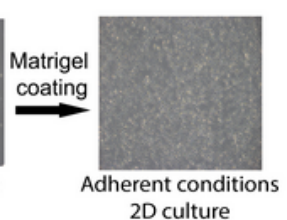

D)

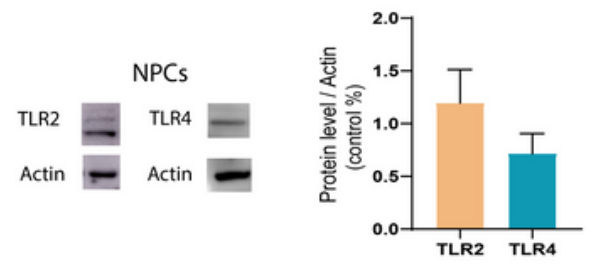

F)

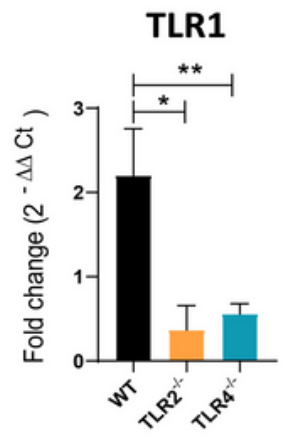

TLR2

TLR3

TLR4
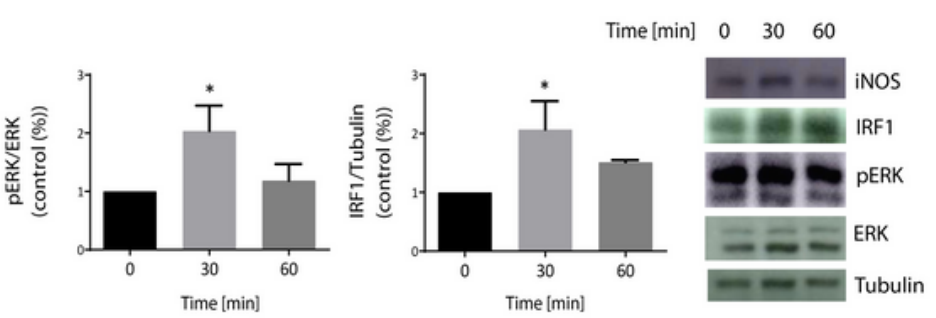

\section{TLR6}
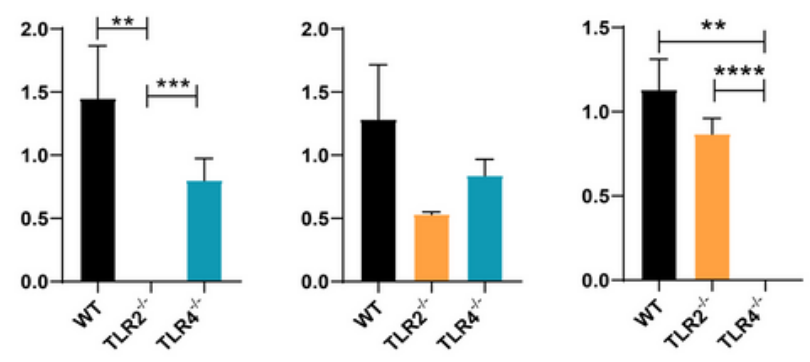

TLR9

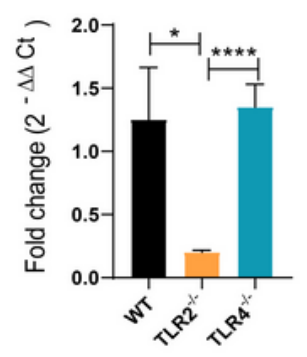

TLR8
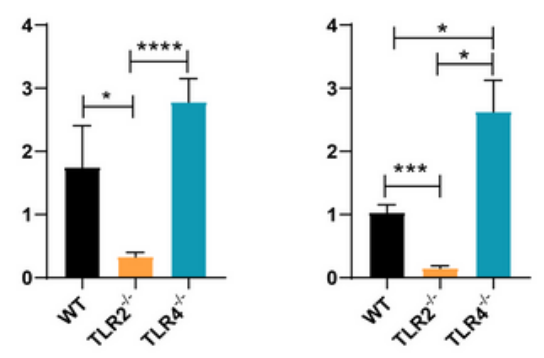

Figure 2

TLR2 and TLR4 Expression Analysis of in vitro-expanded Spinal Cord NPCs. (A) Schematic representation of NPC extraction from neonatal mouse spinal cords and their culture in both neurosphere-like 3D and adherent conditions. (B) Gene expression analysis of TLR2 and TLR4 in WT NPCs. (C) Representative confocal images showing the expression of TLR4 (green), TLR2 (red), and the co-expression (merge) in WT NPCs grown under adherent conditions. (D) Representative Western blot and quantification of TLR2 
and TLR4 protein expression in WT NPCs grown under adherent conditions . E) Densitometric analysis Western blot analysis of iNOS, pERK, and IRF1 levels in response to LPS (50 ng/ml) for 0,30 , or $60 \mathrm{~min}$ in WT NPCs grown under adherent conditions and a representative Western blot. Data shown as mean \pm SEM ( $n=4$ per group) compared with the control group ( $0 \mathrm{~min}$ ) and two-tailed unpaired t-test was used to analyze significant differences; ${ }^{*} p>0.05,{ }^{* \star *} p<0.001$ vs. 0 min.; (F) Gene expression analysis of select TLRs in WT, TLR2-/- and TLR4-/- NPCs. Data shown as mean \pm SEM ( $n=6$ per group $)$ and two-tailed unpaired t-test was used to analyze significant differences; ${ }^{*} p>0.05,{ }^{\star \star} p<0.01$, ${ }^{\star \star \star} p<0.001$, ${ }^{\star \star \star \star} p<0.0001$ as indicated. 

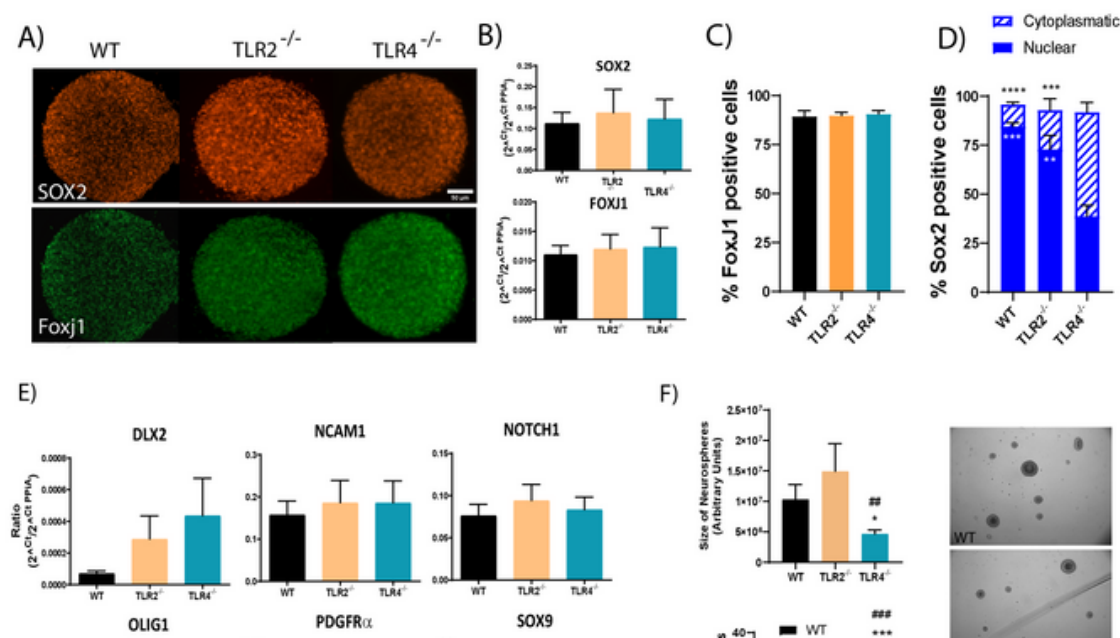

F)
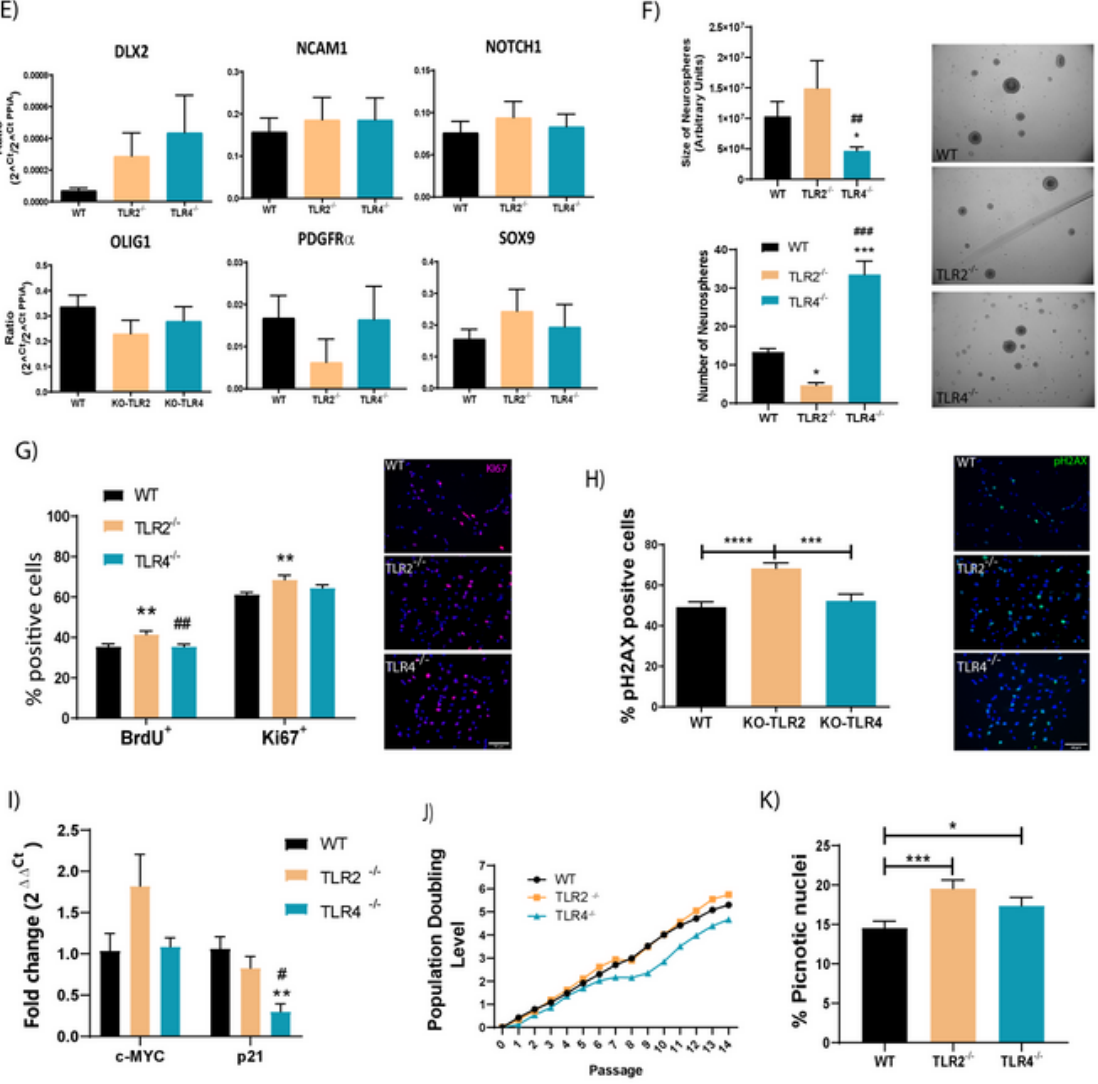

\section{Figure 3}

Involvement of TLR2 and TLR4 in the Self-renewal of in vitro-expanded Spinal Cord NPCs. (A) Representative images of immunofluorescence assay for Sox2 (upper panels, orange) and FoxJ1 (lower panels, green) detection in neurospheres formed using WT, TLR2-/- and TLR4-/- NPCs. (B) Gene expression analysis of Sox2 and Foxj1. (C) Quantification of FoxJ1 positive cells by immunoassay expressed as the percentage of total DAPI positive cells. (D) Quantification of Sox2-positive cells 
represented as nuclear (full) or cytoplasmic expression (striped). Black stars $\left(^{\star}\right)$ vs. TLR4-/-for cytoplasmic Sox2 and White stars $\left(^{*}\right)$ vs. TLR4-/- for nuclear Sox2. (E) Gene expression analysis in WT, TLR2-/-, and TLR4-/- NPCs. (F) Quantification of size (upper graph) and number (lower graph) of neurospheres formed from WT, TLR2-/-, and TLR4-/- NPCs 48 hours incubation after unicellular disaggregation. * vs. WT; \# vs. TLR2-/. Representative images of the indicated neurosphere like cultures are shown (right); (G) (Left) Quantification of BrdU and Ki67 positive cells in WT, TLR2-/- or TLR4-/- NPCs represented as percentages of the total cells. * vs. WT; \# vs. TLR2-/-. (Right) Representative images of the double immunofluorescence - BrdU (red) and Ki67 (green). (H) (Left) Quantification of H2AX positive cells in WT, TLR2-/- or TLR4-/- NPCs represent as percentages of the total cells. * vs. WT; \# vs. TLR2-/-. (Right) Representative images of the pH2AX (green) immunofluorescence with DAPI used for nuclei counterstaining (blue) in WT, TLR2-/- or TLR4-/- NPCs. (I) Gene expression by qPCR of cMYC and p21 transcripts in WT, TLR2-/- and TLR4-/-NPCs. (J) Population doubling level analysis in WT, TLR2-/- and TLR4-/- NPCs expressed as the mean of three independent experiments. (K) Quantification of pyknotic nuclei in WT, TLR2-/- and TLR4-/- NPCs from DAPI nuclei staining (identified as smaller than normal with hyper condensate chromatin) represented as a percentage of the total cells. Data shown as mean \pm SEM. Results were assessed for normality using the Shapiro-Wilk test and one-way ANOVA with Tukey post hoc

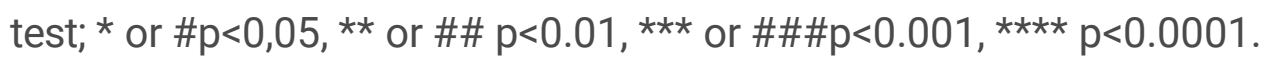


A)
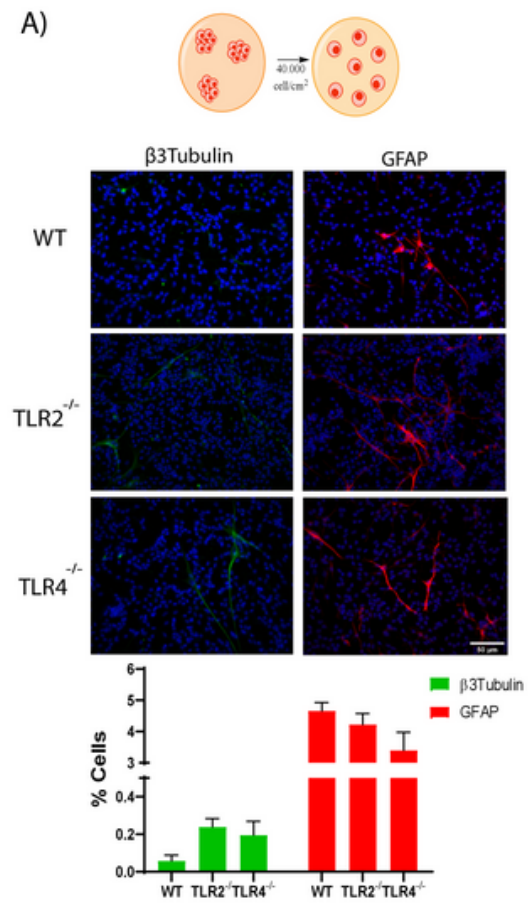

C)
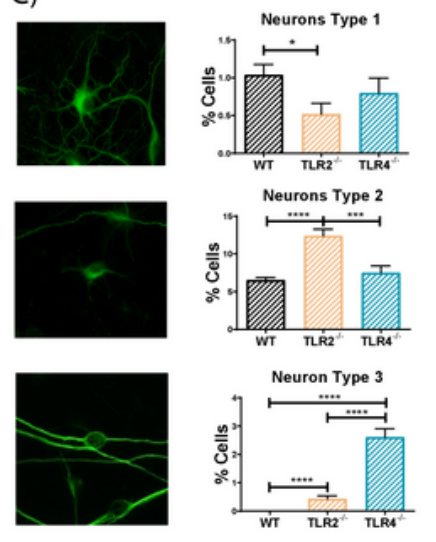

D)

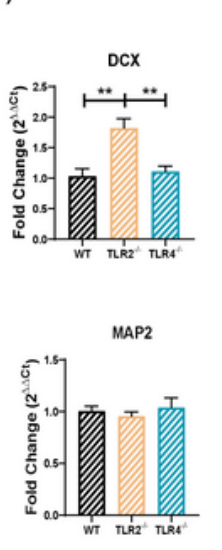

B)

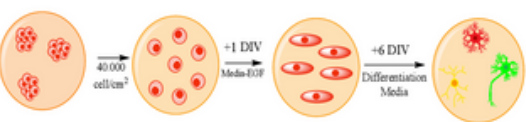

B3Tubulin
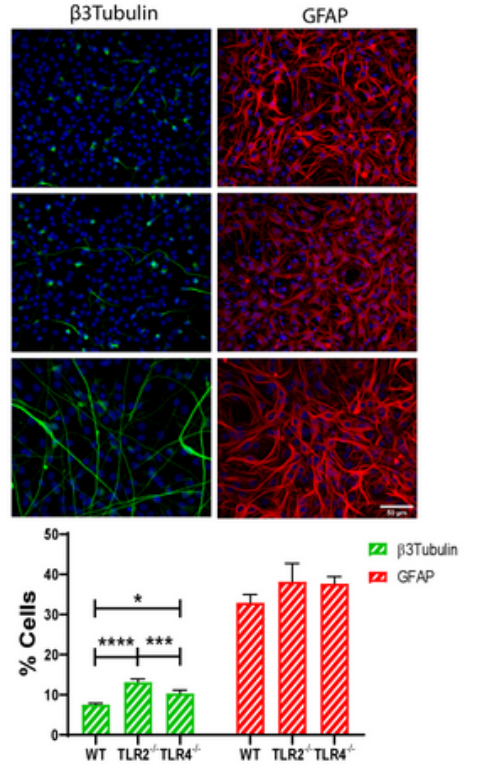

E)

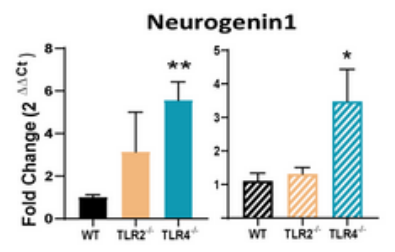

G)
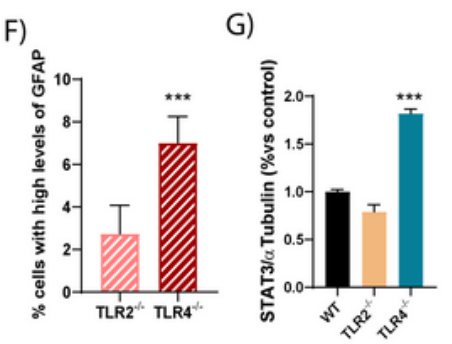

H)

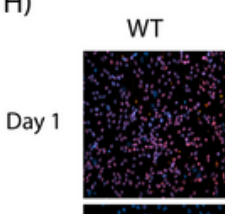

Day 7
TLR2
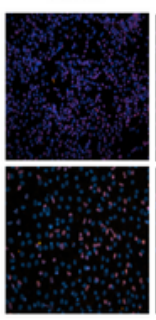

TLR4

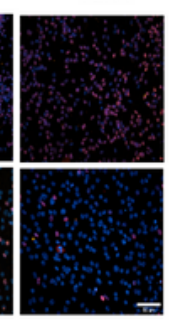

I)

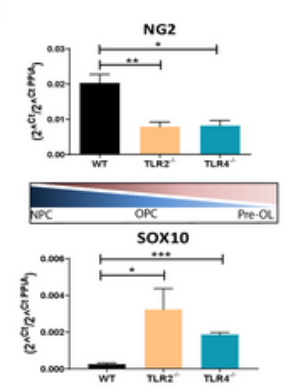

\section{Figure 4}

TLR2 and TLR4 Loss Influence the Spontaneous Differentiation of in vitro-expanded Spinal Cord NPCs. (A, B) Representative immunofluorescence images of $\beta 3$ tubulin (neural marker; green) and GFAP (astrocytic marker, red) and the corresponding quantification data of the percentage of positive cells (lower panels) in NPCs grown in growth medium (A) or in differentiation medium (B) as summarized in the diagrams (top images). DAPI used for nuclei counterstaining (blue). (C) Morphological classification 
of the three distinct types of neurons identified from $\beta 3$ tubulin staining - type 1 (pyramidal-like cells; upper panel), Type 2 (rounded, with no cell expansions; central panel), and Type 3 (bipolar cells; lower panel). Quantification and comparative analysis of the percentage of the corresponding type of neurons shown for WT, TLR2-/-, and TLR4-/- NPCs; (D) Gene expression analysis of Dcx (early neuronal marker, upper graph) and MAP2 (late neuronal marker, lower graph). (E) Gene expression analysis of Neurogenin1 in growth medium (left) or differentiation medium (right) in WT, TLR2-/- and TLR4-/- NPCs. (F)

Quantification and comparative analysis versus WT NPCs of GFAP protein expression levels in TLR2-/and TLR4-/- cells. (G) Protein expression levels of STAT3 in WT, TLR2-/- and TLR4-/- NPCs (a-tubulin as a loading control). (H) (Left) Representative images of immunofluorescence staining of Olig2 (orange) in NPC in growth medium (upper panels, day 1 ) and differentiation medium (lower panels, day 7). DAPI used for nuclei counterstaining. (Right) Quantitative analysis of Olig2 positive cells at one day (upper graph) and seven days (lower graph). (I) Gene expression analysis for NG2 (upper graph) and SOX10 (lower graph). Data shown as mean \pm SEM. Results were assessed for normality using the Shapiro-Wilk test and one-way ANOVA with Tukey post hoc test. ${ }^{*} p<0.05 .{ }^{* \star} p<0.01,{ }^{* \star *} p<0.001$.

\section{Supplementary Files}

This is a list of supplementary files associated with this preprint. Click to download.

- SUPTable1.pdf

- SupplementaryFigure1.tif 